\title{
Biological monitoring of workers exposed to acetone in acetate fibre plants
}

\author{
Akihiro Fujino, Toshihiko Satoh, Tohru Takebayashi, Hiroshi Nakashima, Haruhiko Sakurai, \\ Toshiaki Higashi, Hiroshi Matumura, Haruo Minaguchi, Toshio Kawai
}

\begin{abstract}
Concentrations of acetone in urine, alveolar air, and blood were measured by gas chromatography with flame ionisation detection for 110 subjects occupationally exposed to acetone (mean 372 ppm) in three factories. Significant relations were found between the time weighted average environmental concentration and the concentration in the biological samples. The strongest correlation was between the concentration of acetone in urine and the degree of exposure $(\mathbf{r}=0.71,95 \%$ CI $0.64-0.77)$. This suggests that urinary acetone concentration is the best biological index of occupational exposure to acetone.
\end{abstract}

Acetone is widely used in industry and is considered to be one of the less toxic solvents. It is known, however, that acetone can irritate the mucous membranes of the eyes, nose, and throat because of its high volatility, ${ }^{1}$ and it has physiological effects on the human autonomic nervous system at exposures above $250 \mathrm{ppm}^{2}$ Water/air and blood/air partition coefficients for acetone are extremely high, ${ }^{3}$ but solubility in fat and oil is low. ${ }^{3}$ Most inhaled acetone vapour is absorbed through the lungs and many studies have been made of its pharmacokinetics (uptake, distribution, and elimination. ${ }^{4-10}$ About $20 \%$ is eliminated via the lung and only about $1 \%$ is excreted in urine. ${ }^{10}$

Recently, many researchers have reported significant relations between exposure and urinary concentrations for various solvents. ${ }^{11-16}$ These results

Department of Health Policy and Management, Institute of Industrial Ecological Science, University of Occupational and Environmental Health, Japan A Fujino, T Higashi

Department of Hygiene and Public Health, Tokyo Women's Medical College

T Satoh

Department of Preventive Medicine and Public Health, School of Medicine, Keio University

$T$ Takebayashi, $H$ Nakashima, $H$ Sakurai Japan Industrial and Hygiene Association H Matumura, H Minaguchi, T Kawai suggest that the urinary concentration of some solvents is useful as a biological index of occupational exposure. Although urinary concentration can be used in biological monitoring as an index of exposure to acetone, ${ }^{15}{ }^{17}$ it is not yet clear whether other indices are more appropriate and we know of no field studies on the relation between the environmental concentration and the concentration in urine, alveolar air, and blood.

The purpose of the present study was to investigate the most appropriate biological index of exposure to acetone. We studied workers while at their jobs and quantified the relation between the environmental concentration in the breathing zone and three biological indices of exposure. Using these data, we tried to derive the acetone concentrations in urine, alveolar air, and blood corresponding to the environmental threshold limit value (TLV) as proposed by the American Conference of Governmental Industrial Hygienists (ACGIH) ${ }^{18}$ and the acceptable concentration as established by the Japan Association of Industrial Health. ${ }^{19}$

\section{Subjects and methods}

Figure 1 shows the schedule for sampling environmental acetone, urine, alveolar air, and blood. The subjects were 110 male workers on triple shift operations, aged 18 to 56 (mean 37.6), who worked in three acetate fibre manufacturing plants with different amounts of exposure. All had been exposed to

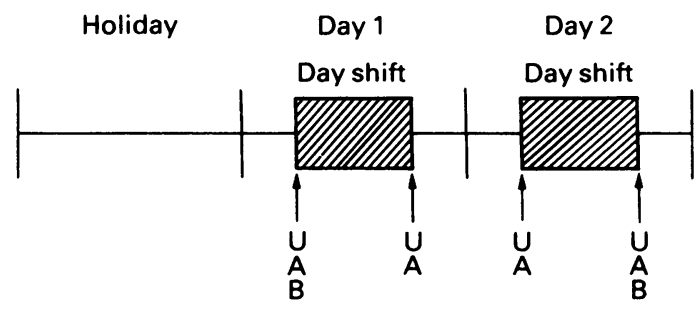

Figure 1 Schedule for monitoring exposure to acetone. The arrows indicate the times at which environmental acetone was measured. The letters indicate collection of urine $(U)$, alveolar air $(A)$ and blood (B) samples. 
Gas chromatographic conditions for measuring acetone concentrations

\begin{tabular}{llll}
\hline & Exposure & Urine & Alveolar air, blood \\
\hline Instrument & GC-Mini 2 (Shimadzu) & GC-15A (Shimadzu) & FGC-110 (Fuji Chemical) \\
Column & Gaskuropack 54 (GL science) & $10 \%$ SBS-100 on Shimalite TPA \\
Column size & $80 / 100 \mathrm{mesh}$ & $60 / 80 \mathrm{mesh}$ & BX100, 60/80 mesh \\
Column temp & $1 \mathrm{~m} \times 3 \mathrm{~mm} \mathrm{ID}$ & $4.1 \mathrm{~m} \times 3.2 \mathrm{~mm} \mathrm{ID}$ \\
Detector & $170^{\circ} \mathrm{C}$ & $60^{\circ} \mathrm{C}$ & $1 \mathrm{~m} \times 3 \mathrm{~mm} \mathrm{ID}$ \\
Carrier gas & FID & FID & $90^{\circ} \mathrm{C}$ \\
\hline
\end{tabular}

ID = Internal diameter; FID = flame ionisation detector.

acetone; no workers were exposed to other solvents. The average duration of exposure to acetone was 14.9 (range 0.5 to 34.3 ) years. Of these subjects, $70.7 \%$ were drinkers and $\mathbf{7 4 . 7 \%}$ were smokers. The physical demands of work were similar in all three factories. Samples of urine and alveolar air were collected before work and within five minutes of the end of work on two consecutive days. Blood samples were collected just before work on the first day and after work on the second day. In total $\mathbf{4 4 0}$ urine and alveolar air samples and 220 blood samples were taken. The amount of exposure to acetone for each subject was measured over eight hours of exposure with a personal passive gas monitor (liquid passive sampler) developed by Minaguchi et al. ${ }^{20}$ This sampler uses water as an absorbent, and does not require desorption to determine vapour concentration.

\section{ANALYSIS OF URINE}

Samples were immediately transferred from paper cups to glass vials and frozen. The acetone concentration in urine (AcU) was measured by directly injecting urine supernatant into a gas chromatograph (GC15A; Shimadzu) with flame ionisation detection. The creatinine concentration and the specific gravity of urine were determined by colorimetry and refractometry respectively.

\section{ANALYSIS OF ALVEOLAR AIR AND BLOOD}

After collection, alveolar air samples were kept at $60^{\circ} \mathrm{C}$ in a constant temperature oven. Blood samples were kept in an incubator at $37^{\circ} \mathrm{C}$ for one hour. The acetone concentration in alveolar air (AcA) was measured by directly injecting alveolar air into a gas chromatograph (FGC-110; Fuji Chemical), and the concentration in blood (AcB) was measured by the vapour liquid equilibrium method with the same gas chromatograph.

DETERMINATION OF THE EXPOSURE CONCENTRATION The time weighted average (TWA) of the concentration of acetone to which each subject was exposed (Acex) was measured with the liquid passive sampler. After sampling, $2 \mu \mathrm{l}$ of the liquid was injected into a gas chromatograph (GC-Mini2; Shimadzu).
The table shows the gas chromatographic conditions for the analysis of $\mathrm{AcU}, \mathrm{AcA}, \mathrm{AcB}$, and Acex.

\section{Results}

The average values for $\mathrm{AcU}, \mathrm{AcA}$, and $\mathrm{AcB}$ before exposure on the first day were $2.44 \mathrm{mg} / 1$ (SD 5.61), $2.95 \mathrm{ppm}$ (SD 7.06), and $3.80 \mathrm{mg} / 1$ (SD 7.07) respectively. These values were considered to be unaffected by other factors, such as drinking alcohol, which contribute to the acetone concentration in samples.

The AcU was strongly correlated with the TWA of the Acex for all the subjects occupationally exposed to acetone vapour on the first day (correlation coefficient $(r)=0.71,95 \%$ confidence interval $(95 \% \mathrm{CI})$ $0 \cdot 64-0.77$ ) (fig 2). We used the uncorrected value for AcU because doing so resulted in a higher correlation coefficient than using the values corrected for specific gravity or creatinine concentration.

A significant relation was also found between Acex

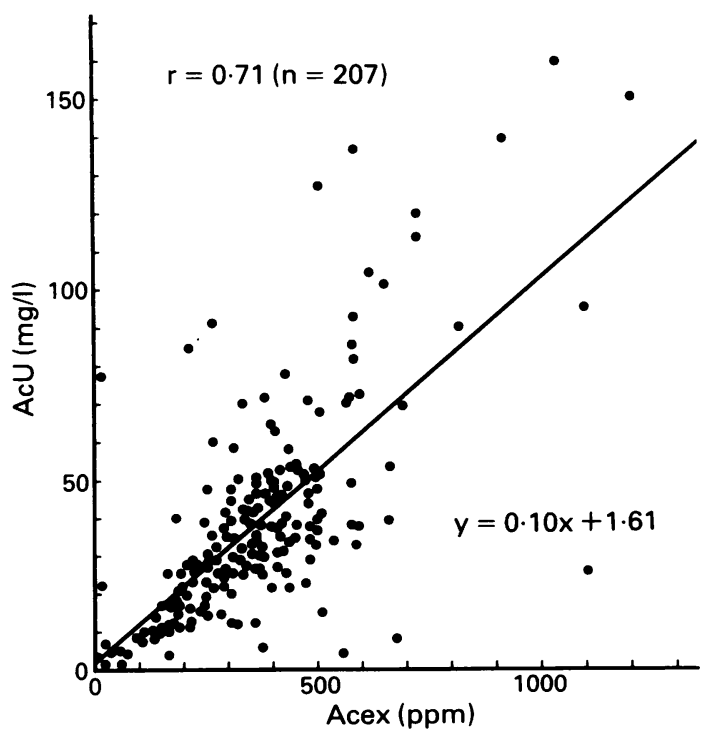

Figure 2 Relation between exposure to acetone (Acex) and acetone concentration in urine $(A c U)$ at the end of work on days 1 and $2(p<0.001)$. 


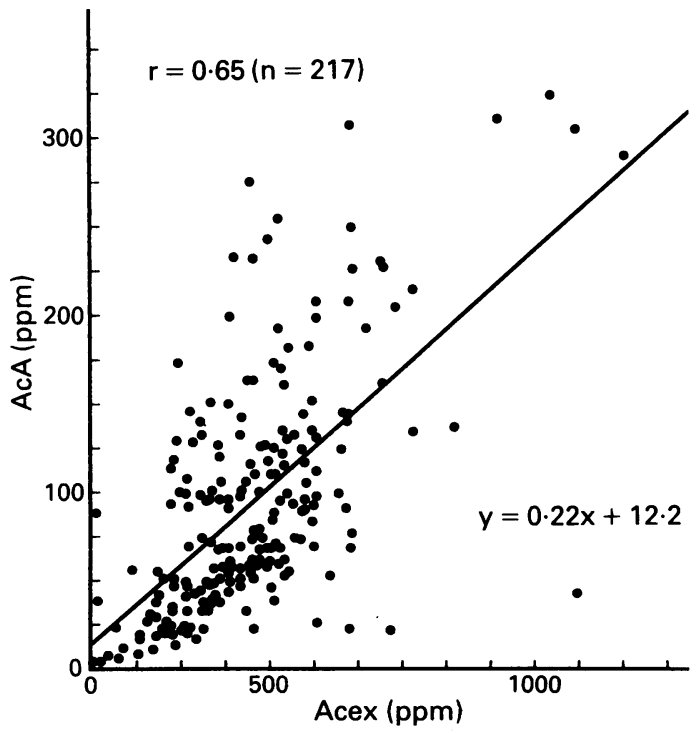

Figure 3 Relation between exposure to acetone (Acex) and acetone concentration in alveolar air $(A c A)$ at the end of work on days 1 and $2(p<0.001)$.

and AcA on the first day $(r=0.65,95 \% \mathrm{CI} 0 \cdot 57-0.72)$ (fig 3).

A close relation between $\mathrm{AcB}$ and the corresponding Acex also existed on the second day of the day shift ( $r=0.65,95 \%$ CI 0.53-0.75) (fig 4).

Also a significant relation existed between AcA and $\mathrm{AcB}$ on the second day $(\mathrm{r}=0.70,95 \% \mathrm{CI} 0 \cdot 59-0 \cdot 78)$ (fig 5).

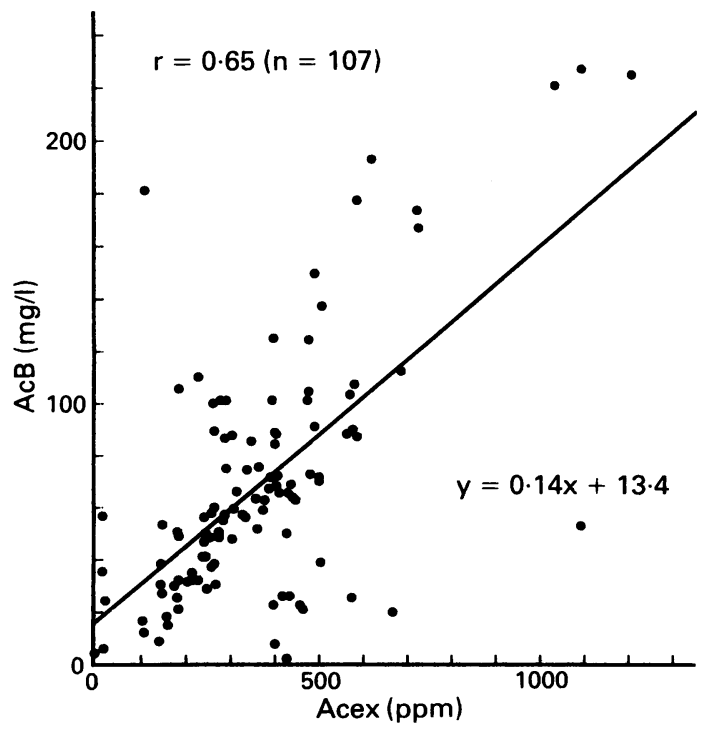

Figure 4 Relation between exposure to acetone (Acex) and acetone concentration in blood $(A c B)$ at the end of work on day $2(p<0.001)$.
The AcU values corresponding to Acex values of $750 \mathrm{ppm}$ and $200 \mathrm{ppm}$ were $76.6 \mathrm{mg} / 1$ and $21.6 \mathrm{mg} / 1$ respectively. The AcAs corresponding to those Acex values were $177 \mathrm{ppm}$ and $56.2 \mathrm{ppm}$ and the corresponding AcB values were $118 \mathrm{mg} / 1$ and $41.4 \mathrm{mg} / \mathrm{l}$ respectively.

\section{Discussion}

In the present study we used samples of urine, alveolar air, and ambient air that were collected on two consecutive days; due to constraints imposed by working in the plants we could only take blood samples on the second day.

Recently, biological monitoring has been widely adopted for the evaluation of amount of exposure. The ACGIH has proposed biological exposure indices, and biological monitoring of several solvents including xylene, stylene, and toluene is required by law in Japan. The possibility of biological monitoring of acetone has been examined in many studies, but such monitoring is not required by law.

The main aim of the present research is to determine the most appropriate biological index of acetone. Therefore, we quantified the relation between Acex and three biological indices of acetone exposure, AcU, AcA, and AcB.

The Acex was closely correlated with all three of these. In particular, the strongest correlation was between $A c e x$ and $A c U(r=0 \cdot 71)$. This correlation did not improve by correcting AcU either for specific gravity of the urine or for the urinary creatinine concentration as found for other solvents. ${ }^{121316}$

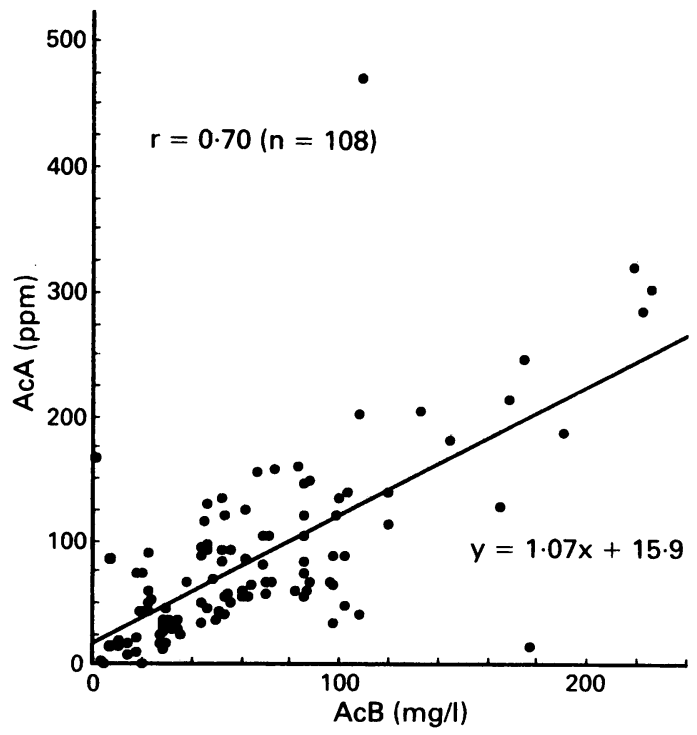

Figure 5 Relation between acetone concentration in alveolar air $(A c A)$ and acetone concentration in blood $(A c B)$ at the end of work on day $2(p<0.001)$. 
Some previous studies have shown a significant relation between TWA environmental concentrations of acetone and AcU, AcA, and AcB, ${ }^{11015172122}$ but all except two ${ }^{15}{ }^{17}$ of those studies were performed in the laboratory. Our results were obtained from many subjects $(n=110)$ after occupational exposure. The present results indicate that $A c U$ is more suitable than AcA or AcB as an index of biological exposure because it was more strongly correlated with Acex. This is probably because urine is continuously produced and accumulated (in the bladder), unlike alveolar air and blood. In fact, there was a close correlation between AcA and AcB $(r=0 \cdot 70)$.

The acetone concentrations corresponding to the TLV (750 ppm) ${ }^{18}$ were $76.6 \mathrm{mg} / 1$ for AcU, $177 \mathrm{ppm}$ for AcA, and $118 \mathrm{mg} / 1$ for $A c B$. The values corresponding to the acceptable concentrations according to the criterion set by the Japan Association of Industrial Health $(200 \mathrm{ppm})^{19}$ were $21.6 \mathrm{mg} / 1$ for AcU, $56.2 \mathrm{ppm}$ for AcA and $41.4 \mathrm{mg} / 1$ for AcB. These values are higher than those given in previous reports. ${ }^{1610}$ Pezzagno et al reported that differences in physical demand changed AcU after experimental exposure. ${ }^{15}$ This may explain some of the difference between our results and those published previously.

We cannot expect a perfect correlation between the Acex and biological indices because of many relevant variables. These include pulmonary ventilation, fluctuation of exposure, physique, metabolism, and alcohol consumption.

In summary, of the three biological indices of acetone exposure examined in this study, the one with the strongest correlation to Acex was AcU. Urine can be collected quickly, the procedures are non-invasive and simple. We therefore believe that the concentration in urine could be the most appropriate indicator of exposure to acetone.

Requests for reprints to: Akihiro Fujino $M D$, Department of Health Policy and Management, Institute of Industrial Ecological Science, University of Occupational and Environmental Health, Japan, 1-1 Iseigaoka, Kitakyushu, 807 Japan.

1 Matsushita T, Yoshimune A, Inoue T, Yamada S, Suzuki H. Experimental studies for determining the MAC value of acetone. Japanese Journal of Industrial Health 1960;11: 477-85.

2 Suzuki $H$. An experimental study on physiological functions of man exposed to acetone gas. Japanese Journal of Industrial Health 1973;15:23-4.

3 Sato A, Nakayama T. Partition coefficients of some aromatic hydrocarbons and ketones in water, blood and oil. $\mathrm{Br} J$ Ind Med 1979;36:231-4.

4 Astrand I. Uptake of solvents in the blood and tissues of man: A review. Scand J Work Environ Health 1975;1:199-218.

5 Astrand I. Uptake of solvents from the lungs. Br J Ind Med 1985;42:217-8.

6 Brugone F, Perbellini L, Grigolini L, Apostoli P. Biomonitaring of industrial solvent exposure in worker alveolar air. Int Arch Occup Environ Health 1980;47:245-61.

7 Egle JL. Retention of inhaled acetone and ammonia in the dog. Am Ind Hyg Assoc J 1973;34:533-9.

8 Hailer E, Filser JG, Bolt HM. Inhalation pharmacokinetics based on gas uptake studies. II. Pharmacokinetics of acetone in rats. Arch Toxicol 1981;47:293-304.

9 Nomiyama $\mathrm{K}$, Nomiyama $\mathrm{H}$. Respiratory retention, uptake and excretion of organic solvents in man. Int Arch Arbeitsmed 1974;32:75-83.

10 Wigaeus E, Holm S, Astrand I. Exposure to acetone: Uptake and elimination in man. Scand J Work Environ Health 1981;7: 84-94.

11 Ferry DG, Temple WA, McQueen EG. Methanol monitoring. Int Arch Occup Environ Health 1980;47:155-63.

12 Imbriani M, Ghittori S, Pezzagno G, Capodaglio E. n-Hexane urine elimination and weighted exposure concentration. Int Arch Occup Environ Health 1984;55:33-41.

13 Miyasaka M, Kumai M, Koizumi A, et al. Biological monitoring of occupational exposure to methyl ethyl ketone by means of urinalysis for methyl ethyl ketone itself. Int Arch Occup Environ Health 1982;50:75-83.

14 Pezzagno G, Ghittori S, Imbriani M, Capodaglio E. Urinary elimination of styrene in experimental and occupational exposure. Scand $J$ Work Environ Health 1985;11:371-9.

15 Pezzagno G, Imbriani M, Ghittori S, Capodaglio E. Urinary elimination of acetone in experimental and occupational exposure. Scand J Work Environ Health 1986;12:603-8.

16 Sedives V, Mraz M, Flek J. Biological monitoring of persons exposed to methanol vapours. Int Arch Occup Environ Health $1981 ; 48: 257-79$

17 Kawai T, Yasugi T, Uchida Y, Iwami O, Ikeda M. Urinary excretion of unmetabolized acetone as an indicator of occupational exposure to acetone. Int Arch Occup Environ Health 1990;62:165-9.

18 American Conference of Governmental Industrial Hygienists. Exposure indices proposed by ACGIH for 1990-1991. Cincinnati, 1984.

19 Japan Association of Industrial Health. Recommended Occupational exposure limits. Japanese Journal of Industrial Health 1990;32:381-401.

20 Minaguchi $\mathrm{H}$, Matumura $\mathrm{H}$, Saito $\mathrm{S}$, et al. A passive gas monitor using liquid for an absorbent (report 4). Proceedings of Association of Industrial Hygiene of Japan 1990;30:75-6.

21 Brown WD, Setzer JV, Dick RB, Phipps FC, Lowry LK. Body burden profiles of single and mixed solvent exposures. $J$ Occup Med 1987;29:877-83.

22 Di Vincenzo GD, Yanno FJ, Astill BD. Exposure of man and dog to low concentration of acetone vapor. Am Ind Hyg Assoc $J$ 1973;34:329-36.

Accepted 2 December 1991 